\title{
TERAPIA NEURAL: LOS DIENTES Y SU RELACIÓN CON EL ORGANISMO
}

\section{NEURAL THERAPY: THE TEETH AND ITS RELATIONSHIP WITH THE ORGANISM}

\author{
Delgado-Álvarez Edwin ${ }^{1}$, Paredes-Andrade Claudio Andrés. ${ }^{2}$ \\ ${ }^{1}$ Dr. Mg. Od. Esp Universidad Católica de Santa María de Arequipa.Perú. \\ 2 Odontólogo de práctica privada. \\ edwinde67@hotmail.com
}

\begin{abstract}
Resumen
El presente artículo, trata uno de los temas poco conocidos por la gran mayoría de los profesionales de la salud bucal, pero de gran interés ya que los problemas que pueden aparecer en las diferentes piezas dentales pueden deberse a los diferentes problemas que sufre un órgano, siendo así un indicador. El objetivo de este artículo es el de abarcar principios básicos de la fisiología neural, siendo el propósito abrir nuevos campos de investigación en cada una de las áreas del servicio de salud bucal.
\end{abstract}

Palabras clave: Terapia, fenómenos fisiológicos, enfermedades.

\begin{abstract}
This article deals with one of the subjects little known by the great majority of oral health professionals, but of great interest since the problems that can appear in the different dental pieces can be due to the different problems that an organ suffers, being an indicator. The aim of this article is to cover basic principles of neural physiology, this the purpose of opening new fields of investigation in each one of the areas of the oral health service.
\end{abstract}

Key words: Therapy, physiological phenomena, diseases.

\section{INTRODUCCIÓN}

Dentro del conocimiento popular hay una frase que dice: "La salud del organismo empieza por la boca", y no está alejada de la realidad, ya que el cuidado inadecuado de los dientes desencadenan problemas de salud, tal es el caso de una caries dental que puede llegar a desencadenar problemas graves de salud a nivel sistémico del organismo, teniendo siempre la mentalidad de ver al cuerpo como un todo, podemos decir que los dientes tienen mucho que ver con la salud de los pacientes y éstos se encuentran relacionados con los órganos y su estado; en el desarrollo del tema se verá la importancia y el verdadero cuidado que debemos tener con ellos. La Odontología Neurofocal tiene una larga historia, En la década de los 50 se nombró por primera vez a este término, por el profesor Voll donde las distintas cargas energéticas de los diferentes órganos producen una reacción en las piezas dentales por medio de un meridiano acupuntural específico, durante el desarrollo de las infecciones en nuestro organismo, se produce una alteración de la polaridad en el campo interferente, cada parte del cuerpo se puede convertir en un campo interferente dando como resultado la presencia del dolor. ${ }^{1,2}$ La terapia Neural es un tratamiento sobre el sistema neuro vegetativo con la aplicación de los anestésicos, sobre todo con la lidocaína y la procaína. ${ }^{3}$ Para que así se pueda neutralizar el campo que se produjo por la presencia de los patógenos.

Son susceptibles a todos los procesos inflamatorios e infecciosos que se producen en el órgano dentario, así como a nivel de las estructuras blandas de la cavidad bucal o maxilares, tales como: pulpitis, gingivitis, parodontitis, alveolitis, osteítis, cicatrices de exodoncia, así como todos los dientes necrosados, la mala posición y los tratamientos defectuosos elaborados. ${ }^{4}$

El objetivo de este artículo es abarcar principios básicos de la fisiología neural ya que es olvidada por la gran mayoría de los profesionales, con el propósito de abrir nuevos campos en la investigación de este tema en cada una de las áreas del servicio de salud bucal. 


\section{ESTADO DEL ARTE}

Generalmente se ha creído que los dientes son partes aisladas en comparación con el resto de órganos que compone nuestro cuerpo, olvidando que el órgano dental forma parte del sistema estomatognático, sin embargo, varios estudios en la actualidad han demostrado que los problemas que se ocasionan en las piezas dentales provoca alteraciones a nivel sistémico un claro ejemplo es el estudio realizado por Lamonte en $2017,{ }^{4}$ donde encontró una marcada relación entre la pérdida de las piezas dentales y las enfermedades cardiovasculares, así como la mortalidad de mujeres de avanzada edad, otro ejemplo claro es la marcada relación que existe entre la uveítis (infección a nivel ocular) y los problemas endo-periodontales según lo encontrado por Peña-Sisto $M$. en la ciudad Santiago de $\mathrm{Cuba}^{5}$ y así podemos citar un sin número de estudios que relacionan los problemas médicos con los problemas dentales, el caso más hablado por los profesionales de la salud dental es el alto riesgo de tener una endocarditis bacteriana por la falta de precauciones durante el tratamiento dental.

Aunque para la gran mayoría de los profesionales de la salud, los dientes no son considerados como parte integral e importante de nuestro organismo dejando a un lado el órgano dental estableciendo que los fenómenos fisiológicos que a él le suceden no ocurren en ninguna otra parte del organismo y al contrario, los fenómenos fisiológicos que suceden en otras partes del cuerpo no pueden suceder de ninguna manera en los dientes. Es como si el diente fuera un sistema cerrado, sin contacto con el exterior.

En ocasiones se tiene en cuenta como factor desencadenante de algunas patologías generales como los estudios citados anteriormente, pero nunca como un elemento con una estrecha relación con las otras partes del organismo. Aunque estos conceptos son acertados desde el punto de vista ortodoxo, hay que recordar el pensamiento sistémico u holístico, que refiere que el cuerpo actúa en conjunto para el desenvolvimiento de las actividades diarias que realizamos, un ejemplo claro y simple es durante el consumo de los alimentos, no pudiéramos asimilar los nutrientes necesarios si no hubiera los dientes para poder triturar los alimentos, siendo este pensamiento indispensable para el desarrollo de este artículo.

El avance de la ciencia nos permite explorar una misma situación en diferentes puntos de vista, es así como han evolucionado los conceptos de salud, enfermedad y terapia, tanto en el sentido general y específico. En nuestro campo tenemos que mencionar a la odontología neurofocal como una herramienta para tener una forma de ver, vivir y sentir la vida de una manera diferente. , $^{6}$

El Cirujano Dentista debe realizar el saneamiento oral para eliminar dichos campos de interferencia y así evitar la propagación de focos infecciosos. Los beneficiados evidentemente son los pacientes que ingresaron a nuestro consultorio para el saneamiento de la patología la cual aquejaba a su bienestar. Nosotros como odontólogos vamos a aliviar y empezaremos no a entender, sino a recordar que los dientes gozan también de las virtudes y posibilidades de reacción a los diferentes estímulos que se le aplica, al igual que cualquier otro órgano de nuestro cuerpo.

Con la Terapia Neural todos los dientes tienen relación con diferentes órganos. La Terapia Neural, es un sistema médico, con una concepción diferente de la causa de la enfermedad, cambiando drásticamente las filosofías de tratar de forma unidireccional a las enfermedades. Citando a Hipócrates que decía: "No existen enfermedades, lo que existen son enfermos". Esta idea tiene una concepción adelantada por completo a su tiempo y sin duda también es revolucionaria en la época actual. Estos diferentes puntos de vista, originaron el nacimiento lo que hoy se conoce como medicinas alternativas, entre ellas se encuentra la Terapia Neural.

Las células de nuestro sistema tienen una carga eléctrica de -40 a $-90 \mathrm{mV}$, las alteraciones en mas o en menos de esta carga en cualquier lugar del cuerpo provocan un trastorno, lo que constituiría una enfermedad. ${ }^{7}$ Con el objeto de procurar la salud, la Terapia Neural, hace uso de una substancia llamada procaína, cuya carga eléctrica es de -290 $\mathrm{mV}$ actuando en las células enfermas provocando que su carga eléctrica alterada o despolarizada se pueda polarizar nuevamente dando como resultado la cura del padecimiento que le afecta, por ejemplo, puede estar en menos de -40 o sobre $-90 \mathrm{mV}$, como la procaína es bipolar, actúa subiendo o bajando las descompensaciones. ${ }^{7}$

Hay una asociación estrecha de varios órganos con las piezas dentales, el grupo de los incisivos se encuentran relacionados con la vejiga y los riñones, así como los dolores referidos sobre todo a nivel de la frente, mientras que a los caninos se asocia con el hígado, los premolares con el pulmón y el colon, por último, los molares se conjugan con el estómago, bazo y el páncreas. ${ }^{8}$ (Figura 1 )

Los terceros molares inferiores están relacionados con los fuertes dolores de cabeza, así también con el corazón, para ello cuando nuestro paciente presenta un cuadro agudo de migraña nosotros podemos realizar la prueba del Test de Huneke; éste método se desarrolla por la aplicación de procaína, lo cual en un principio se dio a conocer como anestesia curativa, su verdadero significado lo obtuvo gracias al fenómeno en segundos de HUNEKE se habla de un fenómeno en segundos cuando después de aplicar inyección en el campo interferente antes llamado foco, en éste caso el tercer molar retenido o semi retenido; se logra eliminar el dolor de cabeza instantáneamente sus efectos a distancia siempre y cuando que ese efecto de alivio, perdure por un determinado tiempo. $^{8}$

Los diferentes dolores en cada pieza dental exteriorizan patologías tales como: gastritis, bronquitis, neumonía, amigdalitis, entre otras. Las piezas dentales no solo indican los problemas que el organismo tiene que soportar, sino puede indicar el carácter del sujeto y esto va ir de acuerdo a la 
REFLEXOLOGIA DENTAL DE LA REPERCUSIÓN PATOLÓGICA QUE ES EJERCIDA SOBRE EL ORGANISMO A TRAVÉS DEL SISTEMA NEURO VEGETATIVO (SITEMA NERVIOSO)

\section{Mediciones verificadas mediante equipos de frecuencia especial de la electroacupuntura del doctor R. Voll - Plochingen - Alemania R. F. Richard Wagner Strasse 5}

Esta lámina ha sido restaurada por el grupo de profesionales que han dedicado el homenaje al Dr. Ernesto Adler.

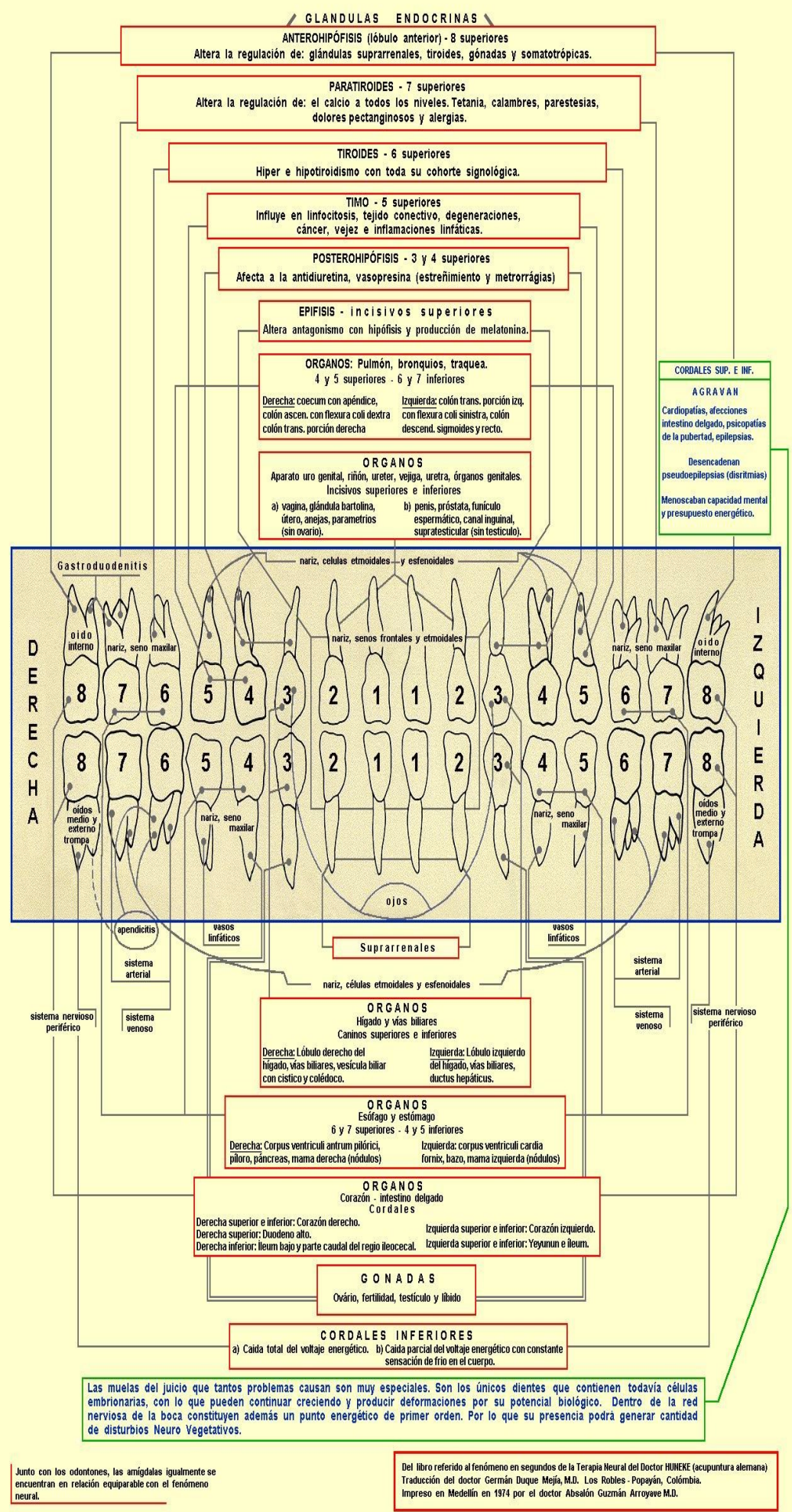

Fig. 1. Asociación de varios órganos con las piezas dentales Revista OACTIVA UC Cuenca . Vol. 2, No. 1, Enero-Abril, 2017 
apariencia de los órganos dentales.

\section{DISCUSIÓN}

Habitualmente los pacientes al acudir a la consulta médica por un gravísimo dolor de cabeza se les receta analgésicos y casi siempre se atribuye a problemas de stress, tensión, algunos lo relacionan con problemas de la vista entre otras posibilidades siendo referidos a otros especialistas; pero jamás lo refieren a un cirujano dentista ya que para muchos y retomando las palabras con las que empecé el artículo dicen erróneamente "¿Qué tienen que ver los dientes con dichas patologías?”.

Refiriéndonos exclusivamente a esta frase, La fisiología médica nos enseña que el sistema nervioso, se encuentra en conexión con todo el cuerpo humano, pero no es una simple conexión, sino un conocimiento profundo de cada una de las partes que lo integran siendo un sistema complejo y termodinámicamente abierto con la capacidad de auto regularse dentro de todo el organismo, ${ }^{7,9}$ es decir, se pone en relación con cada una de sus partes, impulsando tanto al cuerpo, la mente y al espíritu a encontrar un equilibrio y esto se logra a través de la homeostasis, ${ }^{7}$ mostrando que "El todo conoce a las partes y no es que las partes conocen al todo".

Con esto se abrieron caminos totalmente nuevos en la observación de las llamadas enfermedades focales, a través de este fenómeno se pudo establecer la relación entre un determinado mal y su posible origen; como se ha indicado el terreno máxilo mandibular juega un importantísimo papel en el suceso del campo interferente, la estrecha colaboración entre el médico y el odontólogo, es indispensable y se debe establecer un cuadro completo del estado radiológico de toda la boca del paciente inclusive de todos los espacios edéntulos; pudiendo ser una alternativa al tratamiento de la cefalea la exodoncia de los terceros molares. ${ }^{10}$

\section{CONCLUSIONES}

Hay enfermedades que podemos curarlas manteniendo el buen estado de los dientes. La pérdida de cualquier pieza dentaria puede causar alguna interferencia o desequilibrio en la salud general del paciente por tal motivo debemos desvitalizarla. Una buena dieta y una adecuada higiene ayuda a mantener sanos los dientes y por ende nuestra salud en general.

Conflicto de intereses y financiamiento Los autores declaran no tener conflicto de intereses, haber cumplido con los requisitos de autoría y haber autofinanciado este artículo.

\section{Referencias}

1 Chung-Fernández A, Márquez-Zaragoza L, HernándezBorroto C. Relación de la gingivitis crónica con algunas afecciones del sistema osteomioarticular según holograma del microsistema de dientes alemán. MEDISAN vol.17 no.1 Santiago de Cuba ene. 2013.Documento disponible

2 Rojas-Flores C, Reyes Pérez A, Lock-Pérez R, MarreroMartínez J, Muñiz Peláez I, Odontología neurofocal en afecciones ginecológicas. Medicentro 2004; 8(3). Santa Clara -Cuba. Documento disponible.

3 Martínez M, Carbó-Ayala J, Acevedo-Vázquez A. Tratamiento de la glomerulopatía mesangio-capilar con terapia odontológica neurofocal presentación de un paciente, Santa Clara- Cuba, Medicentro 2005;9(2). Documento disponible.

4 LaMonte M, Genco R, Hovey K, Wallace R, Freudenheim $\mathrm{J}$, Michaud D, et al. History of periodontitis diagnosis and edentulism predictiors of cardiovascular diseases, stroke, and mortality in postmenopausal women. Journal of the American Heart Association. 2017;6.

5 Peña-Sisto $M$, Peña-Sisto L, Calzado de Silva M, Rodríguez-Alonso Y, Santos-Toledo L. Focos sépticos bucales en pacientes con uveítis, Santiago de $\mathrm{Cu}$ ba, Cuba MEDISAN 2012; 16(12):1852 Disponible en: Documento disponible.

6 Payán de la Rocha, J. El diagnóstico. Críticas y posibilidades. Terapia Neural, Colombia, 21 de diciembre del 2005 .Disponible en: Documento disponible.

7 Payán I, Monsalvo J. Terapia Neural y Sistema Nervioso".Rev. Terapia Neural, Argentina, 21 Julio 2007 Disponible en: Documento disponible.

8 Nieto M. Cada diente está asociado con los órganos del cuerpo, Rev. esencial natural, 8 Mayo del 2016, Disponible en: Documento disponible.

9 Machiavelli Pelaez R. Qué es la terapia neural, Medicina Neuro-Bio-Energética, Buenos Aires Argentina- 2012. Disponible en: Documento disponible.

10 Payán, J. Aproximaciones al concepto de la salud desde una mirada alternativa. Encuentro Internacional de Terapia Neural en Cuba 2002. Colombia, 27 Abril 2006. Disponible en: Documento disponible. 